\title{
The Application of Infusion Intelligent Monitoring System
}

\author{
Xingjia Jiang, Caihong Yao \\ ${ }^{1}$ Guangxi occupation technology college of machinery and electricity, the department of electric engineering \\ Nanning, China \\ a xingiiajiang@163.com, ${ }^{b}$ ych68@126.com
}

Keywords: the programmable controller; distributed monitoring system; intelligent control; interlock control

Abstract. Aiming at the control accuracy, safety, reliability, low efficiency of traditional manual monitoring infusion system, the introduction of distributed monitoring system is advanced, practical and efficient, implementation of system comprehensive monitoring and management. The PLC application system as a control station, complete infusion state specific measurement and control task. The IPC as the operator station, not only to achieve the monitoring function of infusion system, the pioneering complete audio broadcast scheduling and intelligent infusion control, improve sound alarm and control functions of the infusion system.

\section{Introduction}

Infusion as a basic method to treat diseases, in order to make the process of infusion safety. The patients and nurses need to continuously observe the infusion state, liquid velocity, the infusion time and residual liquid and other information. Therefore, need a infusion automatic monitoring, alarm, control device to improve the safety and reliability of the infusion system and work efficiency. Currently on the market of intelligent infusion device with infusion pump, injection pump type, with perfect function, safety, reliability; but there are also expensive, complex, low degree of limitation of information integration. The distributed monitoring system using classified, hierarchical layout, with centralized management, decentralized control, centralized operation, display and safe, reliable, efficient and practical advantages, at the same time, also provides a basic platform for hospital information system construction and application.

\section{The Distributed Control System Hardware Design}

The overall design of the system. According to the requirements of the work of distributed system and hardware configuration design method, using hierarchical architecture. The system operator station selects the industrial personal computer of very high reliability, the control station chooses the powerful PLC application system, flexible configuration, high reliability. The control station is can work independently, using TCP/IP communication between control and operator station, control station using the PROFIBUS-DP[1].

The control station select Siemens CPU3152DP module as a DP master, ET200M module as the DP the slave station. SM321 digital input module, output module SM322 which belongs to DP the slave station; the communication processor CP343-1 Ethernet communication[1]. When a fault occurs in a control channel, does not affect the operator station of other control channel monitoring. When the operator station or network failure, does not affect the normal work of the control station. In addition, the introduction of redundancy in different levels of the system, further improve the reliability of the system. Integrated infusion of distributed monitoring system is safe and reliable, efficient, practical principles, system hardware structure as shown in Fig.1. 


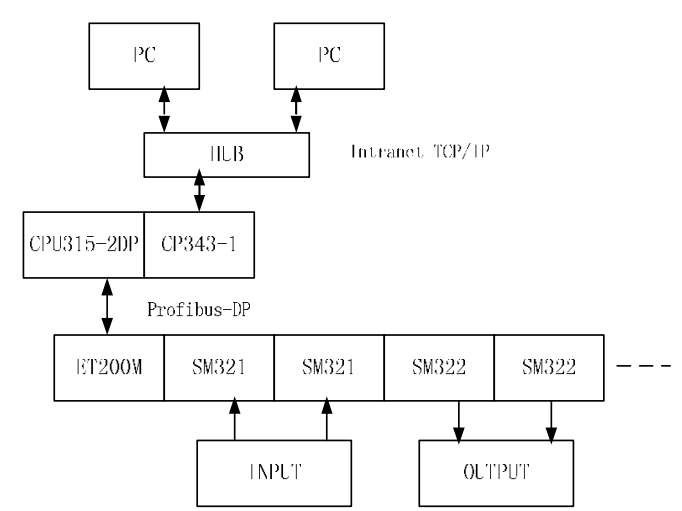

Fig.1 Schematic diagram of the overall structure of the distributed monitoring system

The infusion monitoring device. The infusion monitoring device for field control level, the infusion system can normal work plays decisive role. The infusion monitoring device for detection of the parameters and the output state: liquid level, infusion dripping speed, manual call, nurse confirm, the actuator limit position, the hardware mainly includes the input detection circuit and output control circuit.

The switch input detection circuit. The switch input detection circuit should be correct and reliable to reflect the state of infusion bottle, especially the level; at the same time, taking into account the patients or nursing staff contact the other needs, provide independent call and confirm the channel. The stepper motor drive the gripper reverse movement, control the speed of infusion, the switch is introduced to guide the stepper motor running state for it's limit range of operation. The liquid level detection using two non-contact liquid level switch sensor, a liquid level alarm, another for liquid level alarm; in addition, to improve the infusion speed intelligent control function.

Alarm output control circuit. In order to grasp the current situation, automatically adjust the speed of infusion and guide the follow-up treatment, on the one hand in the operator station and control station to achieve sound and light alarm; on the other hand, the infusion speed adjusting device provides a signal. According to the sound and light alarm, flashing lights from the high level alarm switch pulse periodic PLC to control the digital output port; the operator station interface lights blinking. Sound alarm not only used the PLC buzzer driver, while in the operator station to achieve different beds of the audio signal broadcast scheduling.

Automatic adjustment device of the infusion speed. The infusion rate has some influence on the treatment effect, should be determined the infusion speed, according to the condition of infusion amount, purpose, nature of drugs and infusion stage etc. Routine use of adjustable hand wheel clamp to adjust the infusion speed, there is a certain randomness, especially the completion of the infusion complete can not close causing accidents; therefore, the introduction of intelligent infusion adjusting device.

The main work of the infusion speed adjusting device: the use of infrared photoelectric switch to drip liquid into high and low pulse; in unit time PLC to count on the pulse, to determine the actual drop velocity. The given speed and actual speed based on the control strategy produce the output control; actuator receiving control adjust the infusion process. Because step motor and high ratio of performance to price, execution mechanism of the stepping motor and its driver.

\section{The Control Strategy Analysis}

According to the requirement of process monitoring system, system control strategy can be based on the control station, also be achieved in the operator station. Combined with the characteristics of DCS centralized management and decentralized control, in accordance with the infusion system control strategy of decomposition process. The process control station realize conventional infusion, the operator station control mode to realize the system control strategy and the infusion speed intelligent control algorithm. 
The infusion process mathematical model. The mathematical model of the controlled object gripper opening and dripping speed of inertial lag characteristics [2]: $\frac{k_{1} e^{-\tau s}}{T_{1} s+1}$. A mathematical model of actuator stepper motor and driver for: $\frac{k_{2}}{s^{2}+T_{2} s+k_{3}}$. Infusion of open-loop system belongs to the high order system, on the other hand, due to the factors of change: the medicine liquid volume, the syringe needle and human blood pressure difference. There are characteristics of nonlinear and time-varying and infusion device position and the parameters is difficult to establish accurate mathematical model of system.

The control law analysis. The control law has very important role on the stability of the system, the accuracy and dynamic characteristics, because difficultly establishing accurate mathematical model of the system, the control law to meet the requirements of robustness and adaptability. The PID control strategy is the most widely used in the field of process control, the scaling factor $K_{P}, K_{I}$ integral time and differential time $\mathrm{K}_{\mathrm{D}}$ adjusted to the appropriate value, in order to ensure that the system has good performance. Such as external conditions change, the need to retuning of PID parameters.

Fuzzy control is based on fuzzy reasoning, mimicking the human way of thinking, with the rapid adjustment of the object for difficultly establishing accurate mathematical model, no overshoot, can obtain good dynamic characteristics, but can not eliminate the steady state error. Combining the advantages of both fuzzy control and conventional PID control, fuzzy self-tuning PID control program[3]. Taking into account the stepper motor control the holder operating limit and abnormal state, the holder fully clamped in place, the holder completely released in place, after the completion of infusion, infusion clogging situation, need control algorithm can comprehensively process.

The fuzzy self-tuning PID control system is mainly composed of adjustable PID parameters and fuzzy system two parts, according to the following formula (1) implementation of fuzzy self tuning: $K_{P}=K_{P 0}+\Delta K_{P}, K_{I}=K_{I 0}+\Delta K_{I}, K_{D}=K_{D 0}+\Delta K_{D}$

According to the principle of two-dimensional fuzzy controller, the fuzzy self-tuning PID parameters is to determine the $K_{P}$ varying, the $K_{I}$ varying, the $K_{D}$ varying, according to the error $E$ and error change rate EC fuzzy relation. By constantly testing $\mathrm{E}$ and $\mathrm{EC}$ in the operation, adjusting in real time online the $\mathrm{K}_{\mathrm{P}}, \mathrm{K}_{\mathrm{I}}, \mathrm{K}_{\mathrm{D}}$ three parameters according to the fuzzy control principle, in order to meet the control system requirements in different situations. According to the practical experience and expert knowledge to design a fuzzy controller, the fuzzy self-tuning PID simulation schematic as shown in Fig. 2. Simulation shows that the control scheme of intelligent fuzzy tuning PID has good control performance in the infusion speed.

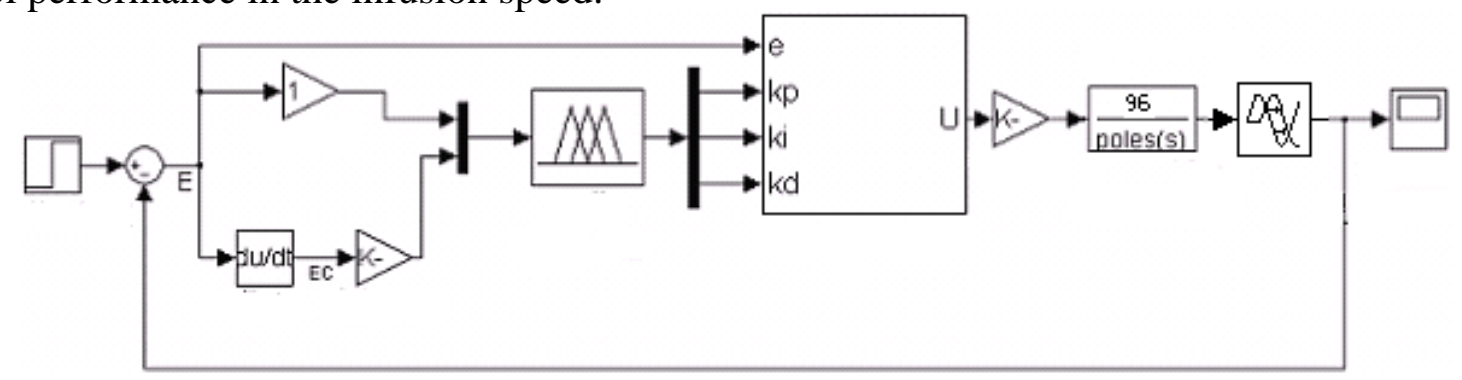

Fig.2 Fuzzy tuning PID simulation schematic diagram

\section{The system software development}

Software development mainly includes the PLC control station and operator station monitoring program, to meet the high performance principles of infusion system, in addition to the operator station alarm audio scheduling, flashing lights, drop speed, the infusion time and auxiliary operation interface, the detection and control of the action is set up on the basis of PLC. The use of PLC ladder 
diagram programming language, combined with the infusion process liquid drop speed, liquid level, the call button, the button state detection and control, development to meet the control requirements of the process of infusion process.

Monitoring software development core modules: configuration interface and application program. The monitoring interface is used to reflect the system process equipment and overall operation, various states, manual operation. In order to improve the performance of the control system, the infusion time, the bed alarm audio dispatch, the infusion speed intelligent control software modules.

The infusion time algorithm. The infusion state despite automatic detection sensor and manual call two means, when sensor and communication networks may be faulty, estimating the infusion time by bottle specifications and dripping speed, improve the medical staff management efficiency. The infusion time algorithm main steps: First, according to the bottle specifications and dripping speed estimate the theory time. Second, read the actual infusion time. Third, compared two time, by the different trend and segmented, showing different state, guiding the follow-up operation.

The alarm audio scheduling algorithm. In order to improve the practicability of the alarm, intelligently scheduling different beds sound alarm display based on shared speaker. The main points of the algorithm: First, all the beds, infusion alarm complete labeling according to the fixed sequence in the data table, and update the contents of the cell in time according to the infusion of the state. Second, read data in a table and bed complete signal alarm audio symbols. Third, such as signal effectively complete the beds, beds for audio identification invalid identifier. Fourth, play the corresponding audio files such as effective audio symbol. Fifth, using the delay mechanism, after completely broadcast the bed alarm audio, cycle retrieve under a bed audio logo.

The infusion speed intelligent control. The fuzzy tuning PID control scheme in the operator station by ActiveX controls to achieve, intelligently tuning PID control by VB development. Not only the realization of fuzzy intelligent control setting core algorithm of PID, providing initial values of the P, I, D parameters, the target value, manual / automatic switch, controller positive action and reaction switching, the real-time trend curve components, comprehensively processing coherent signals. Through properties, methods and events, complying the seamless integration based of the monitoring system configuration software .

\section{Conclusions}

Infusion of distributed system with two working modes of PC monitoring and conventional PLC application system, not only the function perfect and reliable, and has friendly man-machine interface, makes the system flexible and efficient. Complete infusion detection, alarm, control and management under the upper computer supervision and guidance. Adopt multi-effective measures in the system software and hardware, the system has strong anti-interference ability, high safety and reliability, practical features, the actual operation shows that the system has very high practical value.

\section{Acknowledgement}

The 2013 Year Research Project in Guangxi Department of Education to support Project Results "the Application of Intelligent Control Level Monitoring System” (N0: 2013YB298).

\section{References}

[1] Huabo Liu, Wenxue He, Xue Wang, Siemens s7-300/400 PLC programming and application, Beijing: machinery industry press(2010). P.251-255, In Chinese.

[2] Information on http://www.docin.com/p-830412642.html.

[3] Gengyun Yao, Fengxiang Gao, Changsong Wang, et al. Design and Simulation Based on Kalman Filter Fuzzy Adaptive PID Control for Mold Liquid Level Control System, 2009 Chinese Control and Decision Conference(CCDC 2009), GuiLin:IEEE Press, P.6105-6106. 